\title{
Utilizing convolutional neural networks to extract road features from Swiss historical maps
}

\author{
Chenjing Jiao $^{\mathrm{a},} *$, Magnus Heitzler ${ }^{\text {a }}$, Lorenz Hurni ${ }^{\text {a }}$ \\ ${ }^{a}$ Institute of Cartography and Geoinformation, ETH Zürich, Chenjing Jiao, cjiao@ethz.ch; Magnus Heitzler, hmagnus@ethz.ch; \\ Lorenz.Hurni, lhurni@ethz.ch \\ * Corresponding author
}

Keywords: Road Extraction, Convolutional Neural Networks, Historical Maps

\begin{abstract}
:
Historical maps contain abundant geospatial information, such as toponyms, roads, settlements, and hydrology, etc. The hard-copy historical maps are usually digitalized by scanning into raster format for archiving, education ${ }^{1}$ and research purposes (Tsorlini et al., 2014; Heitzler and Hurni, 2020). Among all the geospatial features presented in historical maps, road features are of particular importance, as they have broad application domains, such as transportation (Axhausen et al., 2011), geographical information science (GIS) (Zhao et al., 2015), urban planning (Masucci et al., 2014), etc. Thus, many techniques have been developed to extract road features from raster historical maps to address the intensive demands from these applications (Chiang and Knoblock, 2013; Duan et al., 2020; Saeedimoghaddam and Stepinski, 2020). Nonetheless, these techniques either partly address the goal of road extraction (e.g., to only extract road intersections), or fail in recognizing accurate road boundaries, which calls for more advanced methods (Chiang et al., 2020).
\end{abstract}

We use machine learning methods to segment road features from Swiss historical maps, followed by a vectorization step to obtain the centerlines of the roads. Specifically, we develop a workflow to get training samples from the map sheets, to carry out data augmentation due to the limited amount of training data, to train models using the U-net architecture (Ronneberger et al., 2015) as well as to skeletonize the segmented road areas and vectorise the road lines. Training samples sized 64 x 64 pixels, which cover both road and non-road areas, are automatically clipped from the map sheets. To avoid misclassifying the border pixels, the extent of the training sample is expanded by 32 pixels on each side. The training tiles are sampled intensively around the road features instead of clipping the maps sheet by grids (Jiao et al., 2020) to avoid the unbalanced-data issue as well as to ensure that enough positive samples are obtained. Furthermore, training tiles are also sampled from non-road areas to make sure negative samples are covered. As we only have the labelled road data that cover Zurich city, we apply data augmentation to flip or rotate the training samples, thereby enlarging the training dataset. Then, the training samples together with the labelled road data are fed into U-net. Dice coefficient loss is selected as the loss function, as it outperforms binary cross-entropy loss for imbalanced data (Milletari et al., 2016). The predictions with probabilities larger or equal than 0.5 are segmented as road areas, which are skeletonized and vectorised as road centerlines.

It took around 32 hours to train the model. We generated three-meter buffers for the extracted and the ground-truth road centerlines for computing completeness and correctness, which are $96.69 \%$ and $77.15 \%$, respectively. Figure 1 shows the road extraction results on a subsection of a map sheet, where the red lines present the extracted roads. Figure 1(a) presents an overview of the results, (b) and (c) the zoomed-in parts of urban and suburban areas from (a). It is seen that roads represented by different symbols (e.g., one solid or dashed line, two parallel lines, one thin line together with a thicker line) corresponding to different road grades are well extracted. Future efforts will be focused on classification of the roads represented by different linear symbols, as well as to automatically bridge the gaps in the extracted road lines due to overlaps with other features such as labels or rivers.

\footnotetext{
${ }^{1}$ https://ai.googleblog.com/2020/10/recreating-historical-streetscapes.html
} 

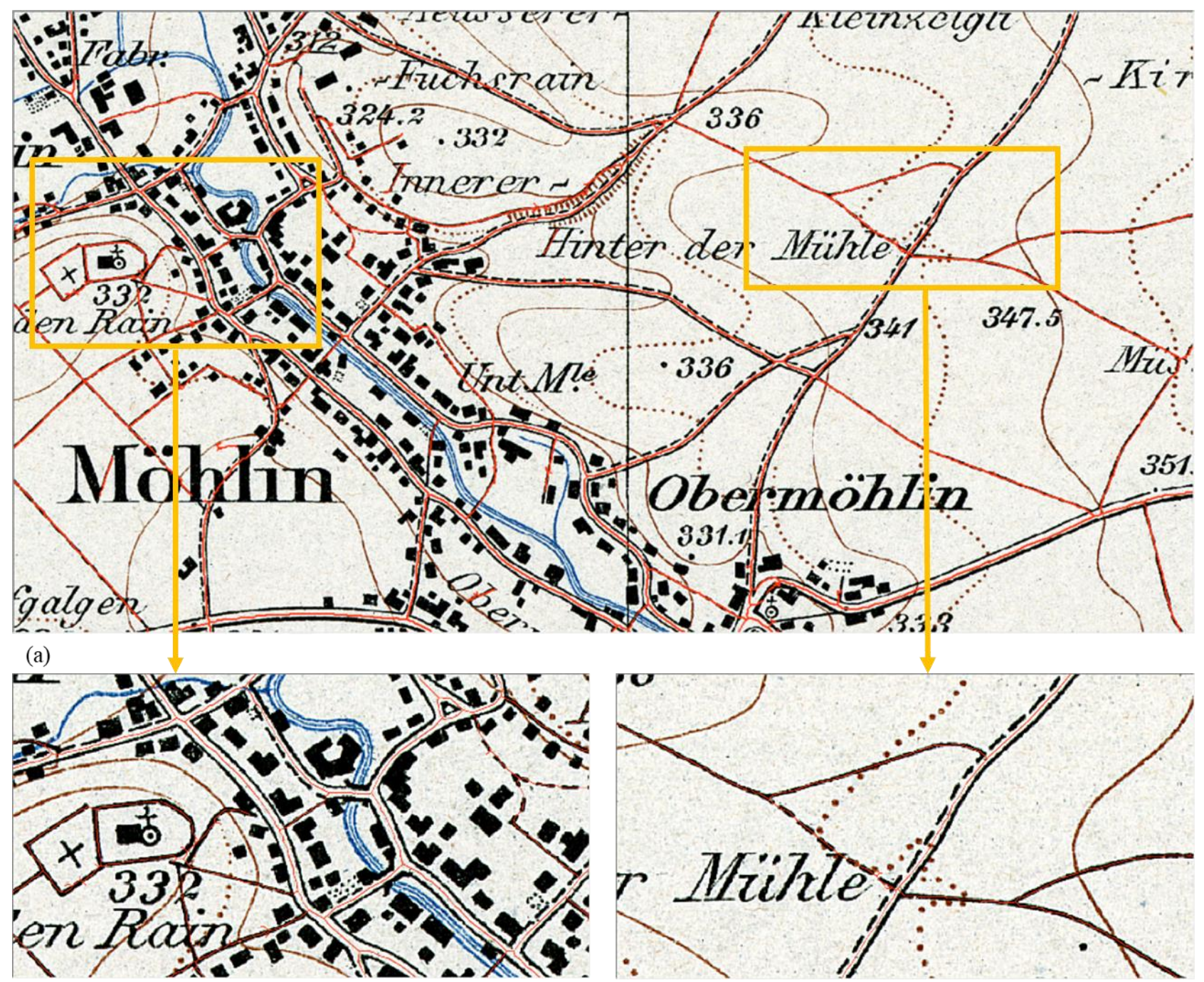

(b)

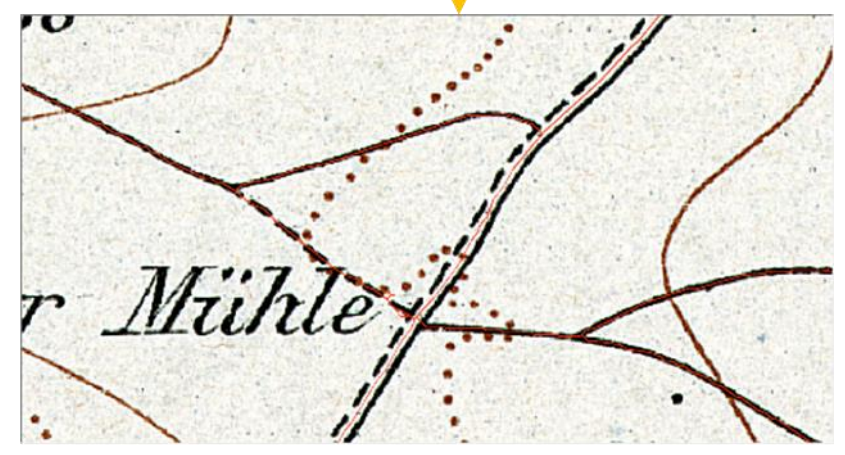

(c)

Figure 1. Road extraction results: (a) an overview of the results on the subsection of a map sheet, (b) zoomed-in part of the urban area, (c) zoomed-in part of the suburban area. 